\title{
حروب الجيل الرابع: الآليات والأبعاد
}

\section{Fourth Generation Warfare: Techniques and}

\section{Dimensions}

\section{شيماء محمد محمد عرفة"}

\section{Shimaa.pr2111@must.edu.eg}

\section{ملخص}

تشهد البشرية اليوم تطورات مذهلة في مجالات علوم الاتصـال وتكنولوجيا المعلومات، وأصبحت تأثيرات هذه التطورات واضحة وبشكل كبير على مختلف جوانـب الحياة الإنسـانية؛ حيث يشهـ العـالم الآن تحـولات كبيـرة في المفـاهيم والنظريات السياسية والعسكرية التقليدية التي سـادت لعقود كثيرة، وذلك بفضل الثورة الهائلة التي أحدثها تكنولوجيا المعلومات والاتصالات، والتي باتت من أهم المحددات المؤثرة في إعادة تثكيل موازين القوى السياسية والعسكرية في العالم، فقد شهد العالم في العصر الحديث تطوراً لطبيعة الحروب واختلافاً في أساليبها عن الحروب التقليديـة السـابقة، وأطلق الباحثون ومراكز البحوث الإسـتراتيجية على هذا النوع من الحروب مُسمى حروب الجيل الرابع. وقد راج مصطلح حروب الجيل الرابع في السنوات الأخيرة لوصف نوع جديد من الحروب غير التقليدية، حيث تُعرف حروب الجيل الرابع بأنها " نـوع من الحروب التي تستهدف القضساء على العدو داخلياً، بـلاً من تدميره عسكرياً * باحثة دكتوراة - قسم الاجتماع - كلية الآداب - جامعة الفيوم. 


\section{باستخدام أسلحة وأدوات مختلفة، والتي تعتمد بشكل كبير على التكنولوجيا"؛ وفي هذا الإطـار سوف تعرض الدراسـة لمفهوم حروب الجيل الرابع من خلال عرض لتطور أجيال الحروب الأبعـة وصـولاً لحروب الجيل الرابع، ومـن ثم الوقوف على أهـم الأليـات والأدوات التـي تقـوم عليهـا، لبنـاء صـورة متكاملـة لملامحها المختلفة، وتوصيف مخاطرها على الأفراد في المجتمع المصري. الكلمات المفتاحية: أجيال الحروب الحديثة؛ الحروب التقليدية؛ حروب الجيل الرابع.}

\section{$\underline{\text { Abstract }}$}

Today, humanity is witnessing amazing developments in the fields of communication sciences and information technology, and the effects of these developments have become clear and largely on various aspects of human life. Where the world is now witnessing major transformations in the traditional political and military concepts and theories that have prevailed for many decades, thanks to the massive revolution brought about by information and communication technology, which has become one of the most important determinants influencing the reshaping of the balance of political and military power in the world. Wars differ in their methods from previous conventional wars. Researchers and strategic research centers called this type of war fourthgeneration warfare.

The term fourth-generation wars has been popular in recent years to describe a new type of unconventional war, where

شيماء محمد محمد عرفة

(حروب الجيل الرابع: الآليات والأبعاد) 
fourth-generation wars are defined as "a type of war that aims to eliminate the enemy internally, rather than destroying it militarily using different weapons and tools, which rely heavily on technology." ; In this context, the study will present the concept of fourth-generation wars by presenting the evolution of the four generations of wars to reach fourth-generation wars, and then stand on the most important mechanisms and tools on which they are based, to build an integrated picture of their various features, and describe their risks to individuals in Egyptian society.

\section{Keywords:}

\section{Modern warfare Generations; Conventional Wars; Fourth-generation warfare.}

\section{أولاً: إثشكالية الدراسة:}

نظراً لمـا تشهـده المجتمعات الحديثة مـن ظـاهرة تتامي استخدام الوسـائل

التكنولوجية بين أفراد المجتمع؛ تبلورت ظاهرة حروب الجيل الرابع والتي اتسمت بطبيعة مختلفة عن نظيرتها التقليدية، من حيث المفهوم والأهداف والخصائص، والآليات والأدوات التي تقوم عليها، وكذلك المخاطر الناجمـة عنها، وبناءً عليه تتطلق إثكالية الدراسة من التساؤل الرئيسي التالي: مـا المقصود بحروب الجيل الرابع من حيث المفهوم والطبيعة، وكذلك الآليات والأدوات التي تعتمد عليها، وأبعاد ومخاطر تأثيراها؟ 


\section{ثانياً: أهمية الدراسةة:}

\section{1- الأهمية العلمية:}

1- سد الفجوة المعرفية المُحيطة بمفهوم حروب الجيل الرابع، من خلال تحديد

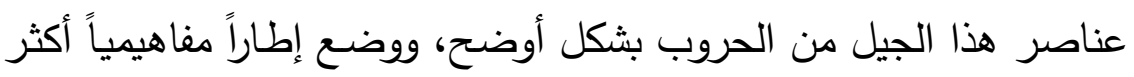

$$
\text { تماسكاً لماهية هذه الحروب. }
$$

2- الكثف عن أهداف حروب الجيل الرابع في المجتمع المصري، والكثف المربف

$$
\text { عن الآليات والأدوات التي تعتمد عليها. }
$$$$
\text { 3- توضيح أبعاد مخاطر حروب الجيل الرابع. }
$$

\section{2- الأهمية التطبيقية:}

1- محاولة وضع منهجية يستعين بها القائمين على وسائل الإعلام في رسم السياسات التي تكفل تقديم محتوى يعزز وعي الأفراد بمفهوم حروب الجيل لهين

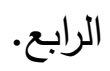

2- يُسهم نشر الوعي بخاطر حروب الجيل الرابع في توجيه صانعي القرار

لسن تشريعات وقوانين خاصة بتلك الحروب.

\section{ثالثاً: أهداف الاراسة:}

1- التعرف على حروب الجيل الرابع من حيث الدفهوم والطبيعة.

2- التعرف على الآليات والأدوات التي تقوم عليها حروب الجيل الرابع.

3- التعرف على أبعاد تأثير مخاطر حروب الجيل الرابع. 


\section{رلبعاً: حروب الجيل الريبع (رؤية بنائية تاريخية):}

لقد تغير مفهوم الحرب وارتبط بهذا التغيير تحولاً حقيقياً في مفهوم أمن الدولة، إذ لم تعد الدولة من حيث هي مؤسسة محلاً للاستهداف من قبل العدو ولا حتى النظـام السياسـي بـل أمتـد الأمـر إلى الكيـان الاجتمـاعي، بغيـة أجراء تعديلات مخطط لها في ثقافة وتصورات الدولة المستهدفة تجعل من استخدام القوة للسيطرة على الآخر أمراً غير ذي جدوى، بل أنه سيؤدي الى نتائج معاكسة مثل المقاومة، والحروب التقليدية وغير التقليدية؛ وتم استبدال هدف الحرب من السيطرة على الأراضي الى السيطرة على العقول، ومن تغيير النظام السياسي إلى تغيير المفاهيم والأفكار ؛ ومن السيطرة المادية المباشرة المؤتتة ذات الكلفة العالية إلى السيطرة على الافراد والجماعات من خلال استهداف مقومات الكيان

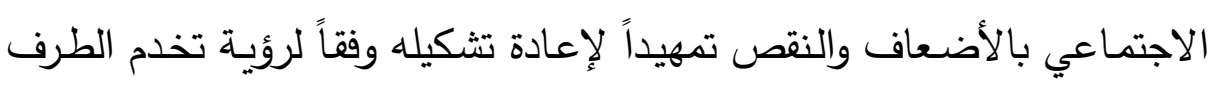
المنتصر في حرب العقول هذه (1).

وتزامنـاً مـع الحركـة المسـتمرة والسـريعة لتطـور تكنولوجيـا المعلومـات والاتصـالات، لم تعد الحروب حروباً عسكرية فقط، بـل أخذت أشكالاً جديدة، واستحدثت وسـائل وأسـاليب عصـرية؛ حيث ظهرت مـا تُعرف بحـروب الجيـل الرابع، لكن قبل توضيح مفهوم حروب الجيل الرابع ينبغي أولاً توضيح الحروب التي سبقت هذا الجيل (2).

\section{• تطور أجيال الحروب:}

الحرب واحدة ولكن تتعدد اساليبها، فهي ظاهرة بشرية قديمة ارتبطت بالنزوع البشري نحو السيطرة من جهة المعتدي وحفظ الذات من جهة المدافع، وستستمر 
بوجود البشرية، ويعكس مفهوم أجيال الحروب ذلك التنوع في وسائل الحرب وما يرتبط بها من غايات تكون هي (مبرر) الحرب، اذ ترتبط الوسيلة من حيث درجة تأثيرها بنوع الغاية التي يراد تحقيقها؛ وإذا كان للحرب معنى واحداً فإن غاياتها، او نطـاق تلك الغايـات (المتمثل بدرجـة الضـرر الذي يُراد أن يلحق يُق بالعدو ومداه الزمني)، هو الذي يحدد الجيل الذي تنتمي اليه حرب معينة من أجيال الحروب (3). حيث تطورت أنواع الحروب إلى عدة أجيال لنُّائم أهدافها؛ وفيما يلي محاولة لتعريف أحيال الحروب وصولاً إلى حوب الحيل الرابع: • مروب الجيل الأول First Generation Warfare: هي حروب تقليدية Conventional Wars بين جيشين نظاميين يمثلون دول في حرب ومواجهة مباشرة وأرض معارك محدة، ويصفها الخبير العسكري "ويليام ليند" بأنها حروب الحقبة من 1648 إلى 1860، وخلال حروب الجيل الأول يتم تنفيذ عدد محدود من العمليات العسكرية، وتشمل عمليات المناورة والالتفاف لتطويـق العدو وضـربه وتـدميره، وتتميز هذه الحـروب بـأن ميدان المعركـة يكون مـنظم فهي التي خلقت ثقافـة النظـام العسكري البيروقراطي، واستمرت حروب الجيل الأول حتى منتصف القرن العشرين، وبدأت في الانهيار مع تطور جيوش العصر الصناعي حيث اصطدمت ثقافة النظام العسكري مع ساحة المعركة المضطربة بشكل متزايد، مما أدى إلى فترة من الارتباك الدموي

في كثير من الأحيان (4). 


\section{" حروب الجيل الثاني Second Generation Warfare:}

يعرفها البعض بحروب العصابات Guerilla Wars، ويعرفها "ويليام

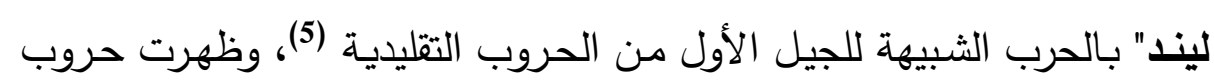

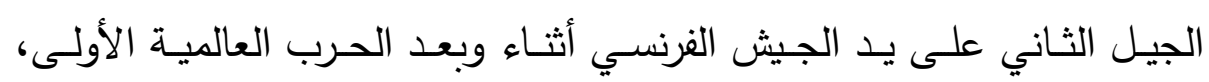
وشهدت تلك الفترة استخدام القوة الناريـة الثـاملة متمثلة في نيران المدفعية، وأصبح الهدف هو استنزاف العدو مـع الحفاظ على ثقافة النظام العسكري

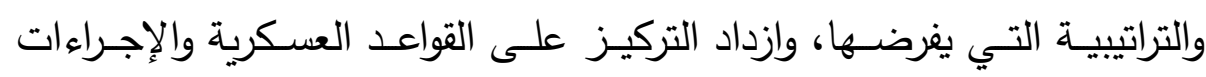

والتعليمات مع طاعة الأوامر (6).

\section{• حروب الجيل الثالث Third Generation Warfare}

تُعرف بـالحروب الوقائيـة أو الاسـتباقية Preventive وهي

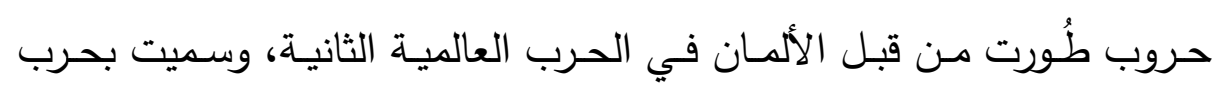

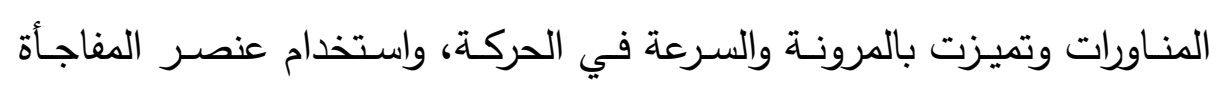
والضرب خلف خطوط العدو؛ وتطورت معها أدوات الحرب فأصبحت تستخدم

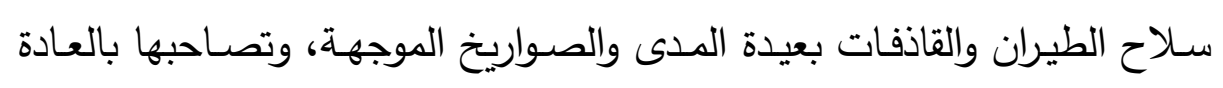
حملات إعلامية مركزة (7).

\section{حروب الجيل الرابع Fourth Generation Warfare:}

يُعد مفهوم حروب الجيل الرابع من الدفاهيم الحديثة نسبياً، ففي أواخر

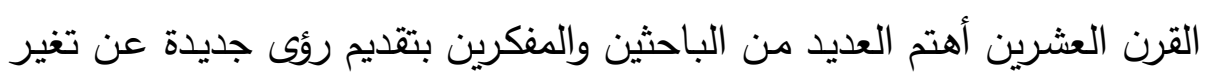

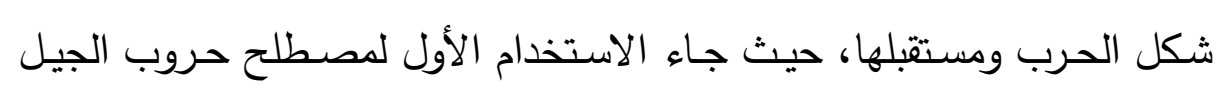

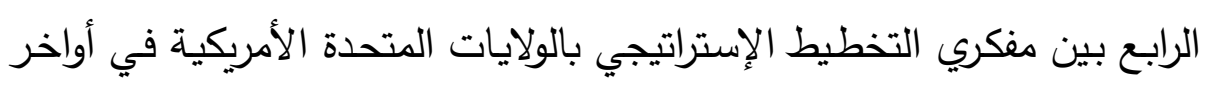

شيماء محمد محمد عرفة

(حروب الجيل الرابع: الآليات والأبعاد) 
الثمانينيات كسبيل لتطوير الديناميات والاتجاهات المستقبلية للحرب، وقد ظهر مفهوم حروب الجيل الرابع في إطـار الجدل الذشهدته هذه الأوسـاط المعنية بإستراتيجيات الحروب كمحاولة للتفكير غير التقليدي؛ وببقى الطرح الأهم الذي أتى بـه الخبير العسكري "ويليـام لينـل" مـع مجموعة من الضباط والأكاديميين العسكريين عام 1989، في مقاله: "الوجه المتغير للحرب: نحو الجيل الرابع"، حيث أرجعوا سبب تغير شكل الحروب إلى التقدم التكنولوجي، وتقلص الحدود الثقافية بين المجتمعات (8). وعندما استقر المفكرون السياسيون والعسكريون الأمريكيون من خلال ورش عمل مختصة بمستقبل الحروب على مفهوم حروب الجيل الرابع، كان تصورهم أن الحرب تدخل بالفعل حقبة جديدة، تتنقل بها من المراحل التقليدية للحروب السابقة التي تعتمد مواجهات مسلحة بين جيوش متحاربة، إلى النفاذ إلى داخل مجتمـع الدولـة المُستهدفة، لإدارة المعـارك في داخلها، فهي معـارك تستخدم مـا يمكن وصـفة بـالقوة الناعمـة، التـي تركزز على تـدمير الإرادة السياسـية للعـدو والتأثير التدميري على عقول صُناع القرار السياسي (9). ويُطلق على حروب الجيل الرابع الحروب اللامُتماثلة أو غير المُتكافئة أو الحـرب ضـــ اللادولـة، حيـث توظـف هـذه الحـروب أنماطـاً صـراعية جديـدة لإخضـاع العدو، مثل الوسائل التكنولوجية المُتتقدمة، والتمرد الداخلي والإرهاب بكافـة صـورة؛ بالإضـافة إلى استغلال كل مـا هو متاح مـن شبكات سياسية واقتصادية واجتماعية وعسكرية ضد الدولة المُستهدفة، ولعل من أهم سماتها، بل أخطرهـا على الأطـلاق هو التمـاهي أو تلاشـي الخطوط الفاصلة بين الحرب 
والسلم، وبين ما هو مدني وما هو عسكري، وبين ممارسـة العمل السياسي وخوض صراع عسكري (10).

وبناءً عليه، يمكن تعريف حروب الجيل الرابع بأنها تلك " الحروب التي لتي

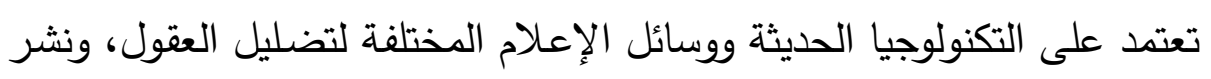

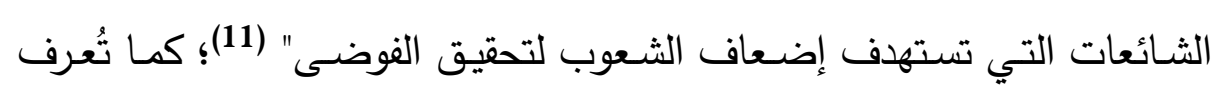

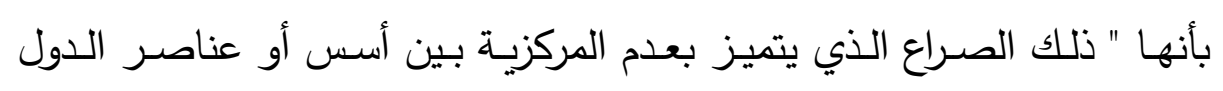

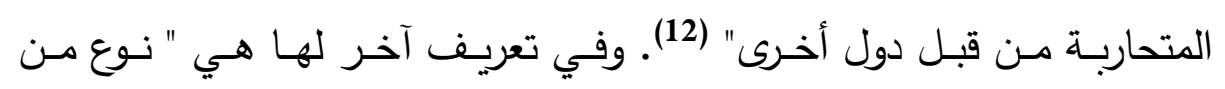

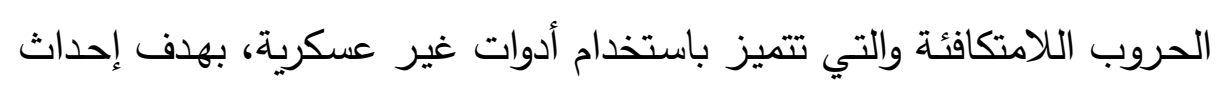
الفوضى والدمار في الدولة المُستهدفة" (13). وبدأ الإعـلام المصري تداول مصـلح " حـروب الجيـل الرابـع " خـلال

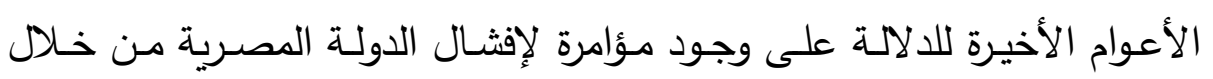

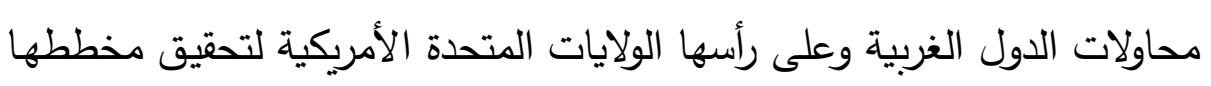

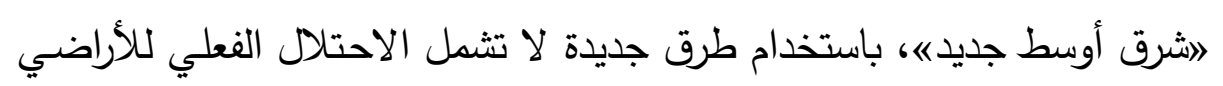

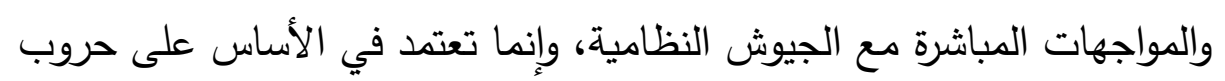
غير متماثلة والتي تستهدف احتلال العقول لا الأراضي (14).

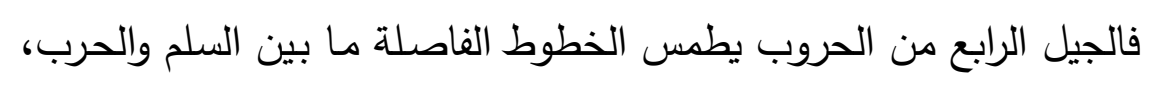

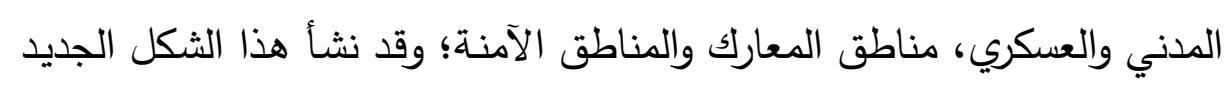

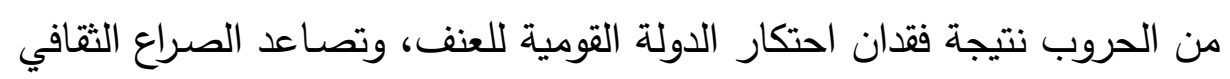
والعرقي والديني، وانتشار العولمة والأساليب التكنولوجية الحديثة على نحو متزايد

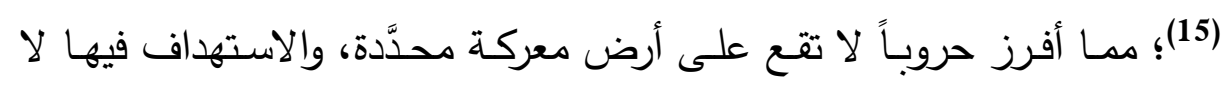


يطول الجنود فقط، وإنما الأفكار، والأطر القانونية، ووسائل الإعلام، والوكالات الدولية، والاتفاقيات، والأشطة الاقتصـادية، والسلطة السياسية، وعقول الأفراد بهدف التدميرين المادي والمعنوي؛ وهنا تتجلى مظاهر مخاطر وتهديدات حروب الجيـل الرابـع خصوصـاً أنهـا تـتم فـي فضــاء مفتوح وواسـع، متجـاوزة الحـدود الجغرافيـة والرسمية، فقد أعـادت هندسـة مفهوم الحـرب بإعـادة هيكلـة الفواعل

والأسلحة، وأساليب الحرب وإستراتيجياتها (16). خامساً: أهداف حروب الجيل الرابع:

1-زعزعة استقرار الدولة المُستهدفة، وشغل جهاز الأمن بها وحرمان القائمين عليه من التمتع بالراحة، وتأجيج الصراعات بين أفراد الدولة المُستهدفة. 2- إحداث حالة من الخوف والهلع والرعب بين أفراد الدولة المُستهدفة، عن طريق الحرب النفسية بالاعتمـاد على وسـائل الإعـلام، والتي تُدار بشكل مُكثف لإحداث هذا النوع من الخلل (17). 3- تفتيـت مؤسسـات الدولـة، والعمـل على انهيارهـا أمنيـاً واقتصـادياً وفكريـاً، وتفكيك وحدة شعبها (18).

4- إنهاك قوة الدولة المعادية والتآكل البطيء في إرادتها، لإجبارها على تتفيذ ما تريده الدول الكبرى التي تستخدم هذا النوع من الحروب، من خلال فرض واقع جديد يخدم مصالحها.

5- إفشال الدولة من خلال عمليات محددة تنفذ في الدول المُستهدفة بخطوات يتم تنفيذها ببطء شديد باستخدام مواطني الدولة أنفسهم. 
6- تحقيق نفس أهداف الحروب التقليدية (الجيل الأول والثاني والثالث للحروب) ولكن بتكلفة مادية وبشرية أقل.

7- تجنب مشكلات مـا بعد الحروب التقليدية مثل الروح العدائية ضد الدولة المُعتدية (19).

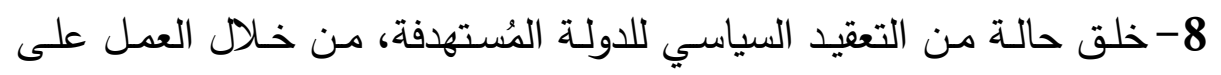

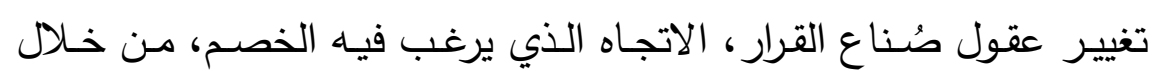
الضغط النفسي والإعلامي (20).

\section{سادساً: خصائص حروب الجيل الرابي:}

الجيل الرابع من الحروب يعني تضاءل استخدام القوة، فالحرب لم تنتهي،

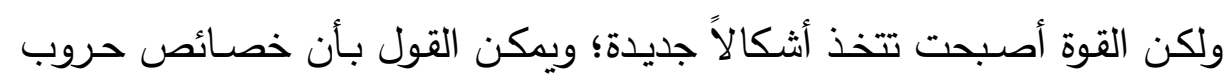
الجيل الرابع تتعدد مـا بين خصائص اجتماعية وسياسية وإعلامية وعسكرية،

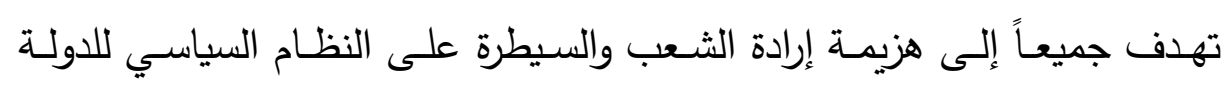
المُستهدفة، وفيما يلي عرض لأهم خصائص تلك الحروب: أولاً: الخصائص السياسية:

1) أنهت احتكار الدولة للحرب، حيث تستغل المسؤولية السياسية للدول تجاه مواطنيها لتطوير إستراتيجيات لإجبارها على انتهاج سلوك سياسي بعينه. 2) شجعت بشكل كبير على ظهور كيانات من غير الدول كالجماعات والقبائل العرقية، والتي تمثل المحرك الرئيسي في تثكيل روح الولاء والانتماء. 
3) أظهـرت الـدور الحاسـم الـي يمكن أن تلعبـه الحمـلات الدعائيـة والحـرب النفسية في التأثير على التوجهات العامة لصانعي القرار.

4) هي حرب طويلة الأمد، ويمكن أن تمتد إلى أجيال حيث تتعدد الإضـعاف المستمر للعدو باستخدام كافة وسائل الضغط المتاحة.

\section{ثانياً: الخصائص الاجتماعية والثقافية:}

5) تعمل على إضعاف التماسك الاجتماعي، وتهديد الهوية الثقافية للمجتمع. 6) تعمل على انخفاض الانسجام في المجتمع، ويصبح العالم المعولم هو البيئة المثالية لتلك الحروب التي تحقق نجاحاً كبيراً في المجتمعات المفتوحة. 7 ) تدهور فكرة الدولة وظهور الصراع الثقافي من خلال بروز حالة من الولاء

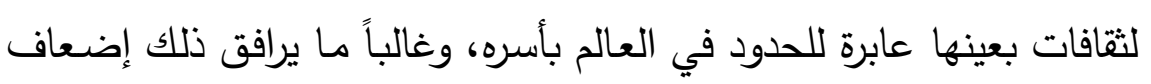
التجانس المجتمعي (21).

\section{ثالثاً: الخصائص التكنولوجية والإعلامية:}

8) اعتمادها على حرب المعلومات التي تعتمد على التقدم التكنولوجي. 9) غياب الطابع المؤسسي فهي تختلف عن الأجيال الأخرى كونها حروباً شبكية حيث لا يوجد لها مركز ثقل يعكس الهيكل المؤسسي.

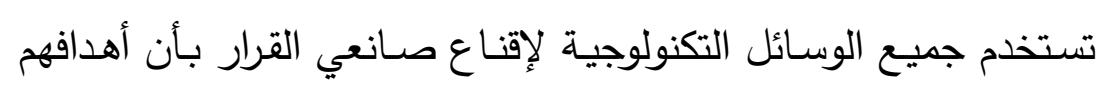
الإستراتيجية غير قابلة للتحقيق. 


\section{لإيعاً: الخصائص العسكرية:}

تعمل على طمس الخطـوط الفاصـلة بـين الحـرب والسياسـة، والصـراع

والسـلام، والجنود والمدنيين، والعنف في سـاحة المعركة والمنـاطق الآمنـة،

مما يفسر الضبابية الثديدة الذي تُميز هذا الجيل من الحروب.

تحويـل الدولـة المُسـتهدفة مـن حالـة الدولـة الثابتـة (القويـة) إلى الدولـة

الفاشلة (الهشـة)، فهي حروب لا تستهدف الجنود فحسب، ولكن تستهدف

$$
\text { الدولة بالكامل بما فيها من مدنيين. }
$$

منتشرة في جميع أنحاء العالم، وليس لها ساحة معركة محددة (22).

\section{سابعاً: آليات (أساليب) حروب الحيل الرلبع:}

لمّا كانت حروب الجيل الرابع حروب غير تقليدية، فمن البديهي أن تستخدم تكتيكات مُغـايرة في طبيعتها عن تلك التي اعتدنا أن نشـاهدها في الأجيال السابقة من الحروب، فهي تعتمد لا على المواجهة المباشرة بين الجهات المُعادية والدولـة المُسـتهدفة، ولا نشـهـ فيها قتال بين جيثـين نظـاميين بمفهوم الحرب التقليدي، ولكنها تعتمد على آليات وأساليب أخرى أكثر ضراوةً وتأثيراً على الدول المُستهدفة (23)؛ وبالنظر إلى مـا شـهـته مصر عبر السنوات الأخيرة يتين لنـا أهم الآليات المُستخدمة لتحقيق أهداف تلك الحرب فيما يلي: 1 - الإرهاب:

حيث يعتبر الإرهاب أحد أهم الأساليب الرئيسية في حروب الجيل الرابع،

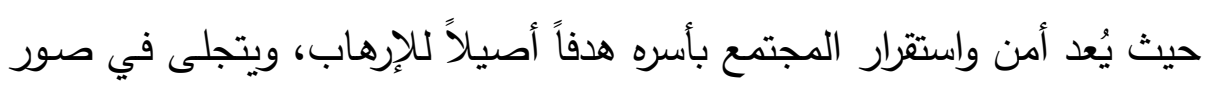


عديدة منها: الاعتداء على المنشئات العامـة والخاصـة، والتركيز على إحداث الفتن الطائفيـة، وكذلك ممارسـة العمليـات الإرهابيـة، حتى يمكن القـول بـأن الإرهـابيين هـ اليد العليـا والفاعلـة في حروب الجيل الرابع (24)؛ كما أصبح الإرهاب الإككتروني أكثر صور الإرهاب شيوعاً في الآونة الأخيرة، وذلك نظراً

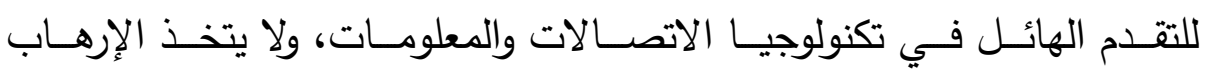
الإكتروني شكلاً واحداً وإنما تتعدد أشكاله وتتتوع صوره وأساليبه، وتتمثل أشكاله في التجسس الإكتروني، والقرصنة على المواقع الحيويـة للمنشآت والمؤسسات الرسمية في المجتمعات المختلفة، والتجنيد الإلكتروني وغيرهـا، كما أن أدواته متعددة مُتمثلـة في اختراق الفيروسـات للبيانـات وتـدميرها، والتجسس وتجنيــ الإرهابيين، وجمع الأموال وتمويل العمليات الإرهابية، وحروب الدعاية والأفكار المتطرفة والهدامة وغيرها (25).

\section{2-حرب الثائعات (حرب المعلومات):}

تعتبر الثائعات أقوى أسلحة حروب الجيل الرابع، حيث تتعدد الغايات التي تقف وراء حروب الثـائعات بحسب الأطراف التي تقودهـا، وتتعدد وفقاً لذلك صورها، فهناك مجموعة متداخلة من أهداف حروب الثائعات التي لا تخرج عن كونها أهداف هدَّامة ومدمرة تمس جميع جوانب الحياة؛ أهمها الأهداف النفسية من خـلال التأثير على الروح المعنويـة وتفتيتها وتدميرها، وأهداف اجتماعيـة بغرض إثارة الفتن والخصـومات وتعميق الخلافـات القائمسة بـين بعض فئـات

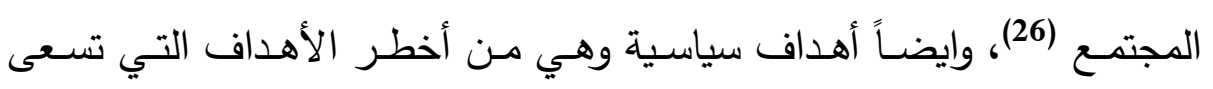
الشـائعات إلى تحقيقها، وذلك بما تتتاولهـ من تشويه لقادة الأمـة والشخصيات 
البارزة فيها؛ وهناك أهداف أخرى لحروب الثـائعات منها بـث الخوف والحقد والكراهيـة والعـداوة بـين صـفوف الثـعب، وتحطيم إرادة العـدو، وتهبـيط الـروح المعنوية للمدنيين والعسكرين، وكذلك تعبئة الرأي العام أو تضليله حول موضوع ما. وتعتبر الثائعات الفكرية من أهم أصناف الثائعات، والتي تستهدف الأمن الفكري الذي هو ركيزة كل أمن وأساس لكل استقرار ، ويُعد هذا النوع من أخطر أنواع الثائعات وأثدها تأثيراً (27). حيث تقوم حروب الثـائعات على بـث المعلومـات الخاطئة عن الأحداث ونشر الأخبار المزيفة ضد رموز الدولة المُستهدفة وضد المشروعات التتمويـة والخطط التوسعية التي تقوم بها الدولة، بهدف افتعال أزمات تثير الرأي العام وتُشـعره بعدم الأمـان، وتدفعـه إلى مواجهات مـع صـانعي القرار السياسـي في الدولة بهدف ألا يلتف الثعب حول فكرة الدفاع عن الأمن الوطني، والتصدي لأي غزو فكري لإفشال الدولة، وتعتمد حروب الشائعات بصورة جوهريـة على وسـائل الإعـلام التقليديـة والجديدة وخاصـة وسـائل التواصل الاجتمـاعي، وذلك لانتشـار تلك الوسـائل وتوافرهـا لدى جميع أفراد المتهـع بمختلف فئاتـه، مــا يُسهل وصـول الثـائعة إليهج، وتأثيرهـا البالغ في نفوسهم (28)؛ وتستخدم ايضـاً الأفلام والدراما التليفزيونية الجاذبة لتغيير أفكار وعادات وتقاليد الشعوب تمهيداً لتغيير الهويـة الفكريـة والثقافيـة لـديهم ضــن اسـتراتيجية الهيمنـة الفكريـة التـي تعتمدها حروب الجيل الرابع (29). 


\section{3-ضرب البنية التحتية للدولة:}

يُعـد ضـرب البنيـة التحتيـة للدولـة المُسـتهدفة واحداً مـن أهـم الطـرق التـي

تستخدمها حروب الجيل الرابع، وذلك من خـلال زعزعـة الاقتصـاد، واستهداف المرافق الحيويـة كمطـات الميـاه والكهربـاء وخطـوط الغـاز، واستهداف بعض

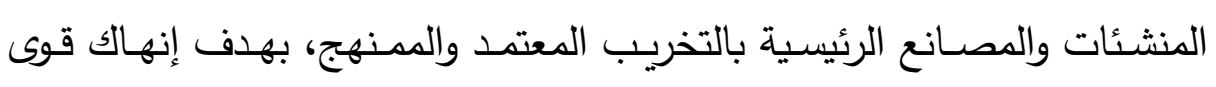
الدولة وإسقاطها من الداخل (30). 4 - الحرب النفسية:

من أهم أساليب حروب الجيل الرابع الحرب النفسية المتطورة من خلال الإعـلام والتلاعب النفسي، باستخدام محطات فضـائية تتشر الأكاذيب وتزور الصـور والحقـائق، وتسـتخدم فيهـا وسـائل الإعـلام التقليديـة والجديـدة (وسـائل التواصل الاجتماعي)، والتي تهدف إلى تضخيم التحديات والتهديدات التي تواجه الدولة المستهدفة، وزرع عدم الثقة في نفوس المواطنين، لإقناعهم بعدم قدرتهح على مواجهـة هـذه التحديات، بالإضـافة إلىى قطـع التواصـل بـين المـواطنين وقياداتهم على كافة المستويات من خلال التشكيك المستمر في أدائهم وانجازاتهم (31). وتُعـد التكنولوجيـا والإعـلام أحد أهـ الأدوات الفعالـة في هذا النمط مـن الحروب، بهدف تشويش عقول أفراد المجتمع ممـا يحقق حالـة من الاستجابة التلقائية لكل ما يتلقونـه من معلومـات، والتتي تكون مناهضـة للدولة ومؤسساتها مما يساعد على خلق حالة من الاستياء الجماعي تجـاه الدولة بهدف زعزعـة أمنها واستقرارها. 


\section{5- 5 إثارة الفوضى:}

حيث تعتمـد هذه الحروب على محاولـة إثــال الدولـة المستهدفة بثورات وأحداث شغب وتمرد واعتداء على المنشآت العامة والخاصـة؛ حيث تتوالى هذه

الأحداث بشكل متسارع يصعب معه السيطرة عليها (32). 6-تجنيد بعض الأفراد والهيئات:

حيث تعتمد هذه الحروب على تحريك جماعات وتتظيمات داخلية مدربـة ضـــ النظام القائم في الدولة المُستهدفة مثل استغلال بعض منظمات المجتمع المـني، وبعض الأحزاب السياسية وكذلك بعض الناشطين مـن دعـاة الحريـة والديمقراطيـة، ومحاولـة افتعـال مواجهات بين قوات النظـام ومؤسسـاته الأمنيـة وعناصـر هـذه الجماعـات، واسـتدراج هـذه العناصـر لارتكـاب خروقـات أمنيـة وتجاوزات تتتافى مع المواثيق والأعراف الدولية الخاصـة بالحريات العامة وحقوق الإنسان (33)

\section{ثامناً: أدوات (وسائل) حروب الجيل الرلبع:}

تتعدد الأدوات والوسائل التي تعتمد عليها حروب الجيل الرابع وفقاً لأهمية الهدف الذي تقوم لأجله هذه الحروب، والمجال الذي تتوي استهدافه عسكرياً، أو سياسياً، أو اجتماعياً، أو اقتصـادياً، أو فكرياً وثقافياً؛ وانطلاقاً مـن هذا يمكن حصر أهم أدوات حروب الجيل الربيع فيما يلي: 


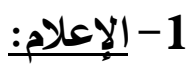

يُعد الإعلام بمختلف صوره أقوى أسلحة حروب الجيل الرابع على الإطلاق،

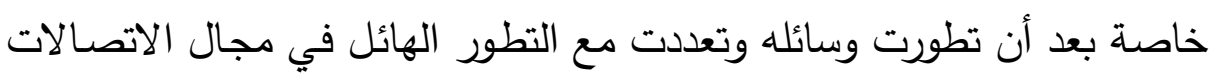
وتكنولوجيا المعلومات، من الصحافة المطبوعة وحتى شبكة الإنترنت ووسائل

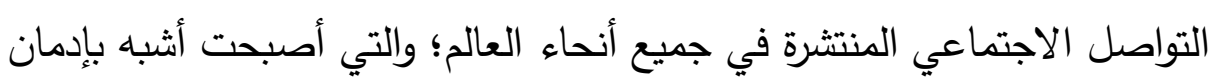

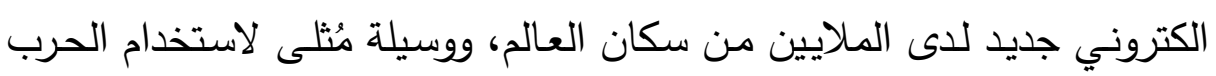

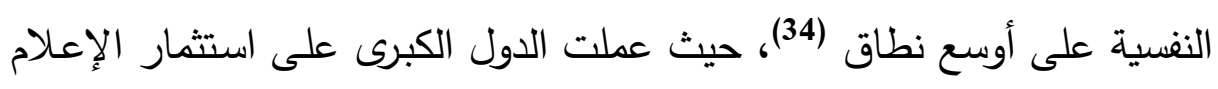

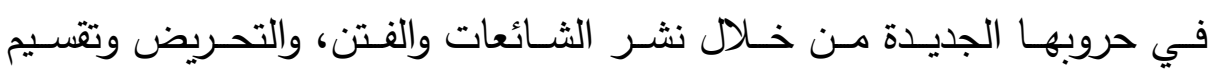

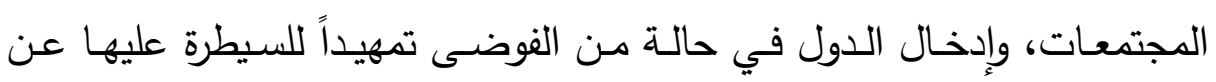

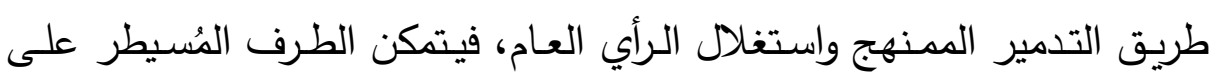

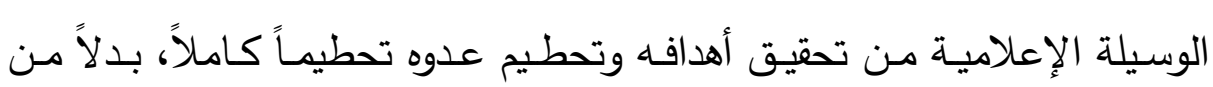
اللجوء إلى الحرب العسكرية المباشرة وما ينتج عنها من خسائر مادية وبشرية.

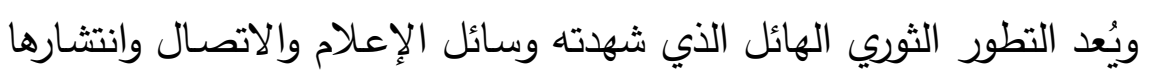
العالمي، وخاصة الإلكتروني منها والتي تتميز بسهولة استخدامها وتوفرها بشكل

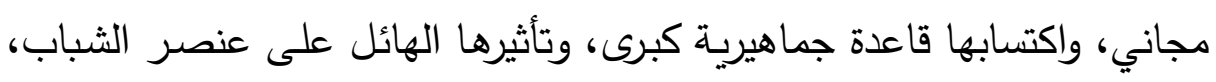
أصبحت هذه الوسائل من أهم عوامل التغيير الثقافي والاجتماعي والسياسي (35)؛ وازدادت الأهمية التي تلعبها شبكات التواصل الاجتماعي في هذا الصدئ نظراً لتميزها بسهولة الاستخدام وسرعة الانتشار ومساهتها في نشر الأفكار عالمياً، من خلال توفير مادة معلوماتية والتي قد تكون مغلوطة لتوجيه أذهان الجمهور نحو قضية ما وكسب التعاطف لصالح طرف بعينة (36).

شيماء محمد محمد عرفة (حروب الجيل الرابع: الآليات والأبعاد) 
كما أصبح الإعلام الجديد يُمثل جوهر أدوات القوة الناعمة والتي حلت محل

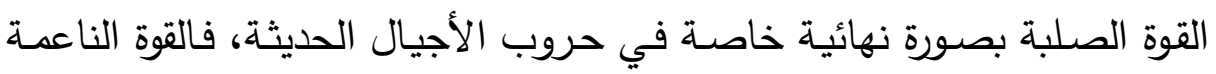

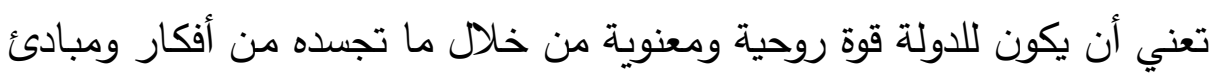

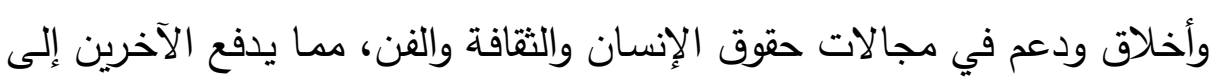

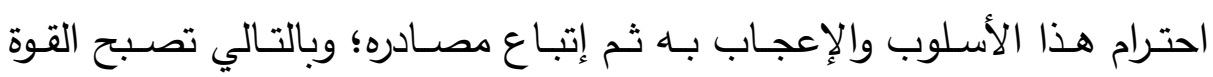
الناعمة من أفضل الأدوات السياسية والعسكرية، إذ تستطيع السيطرة على الأفراد من دون أي تدخل عسكري (37).

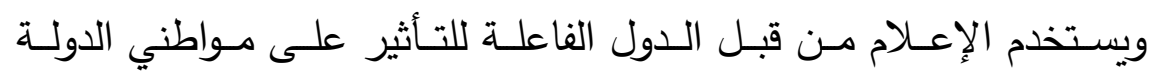

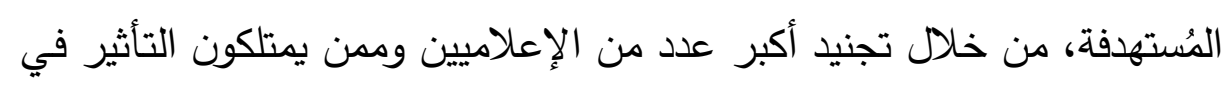

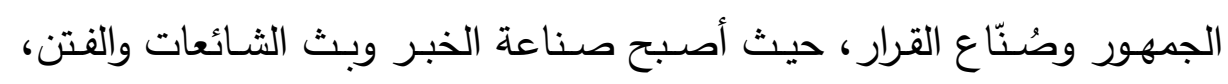
وتضخيم أحداث بعينها وتجاهل أخرى أمر يسير ، ويُستغل الإعلام في التأثير

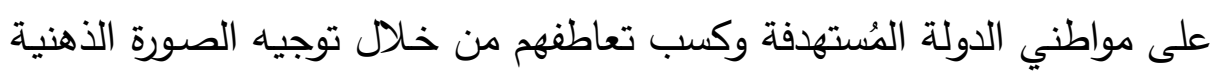

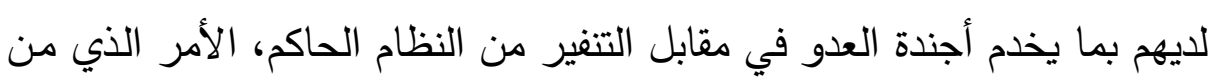

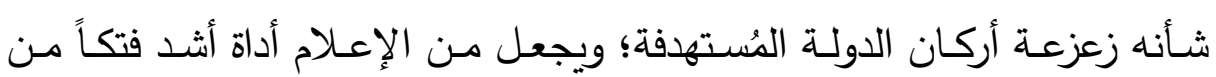
الجيوش العسكرية (38). 2- أجهزة الاستخبارات:

حيث تمتاز حروب الجيل الرابع بتركيزها الثديد على الدور الاستخباراتي

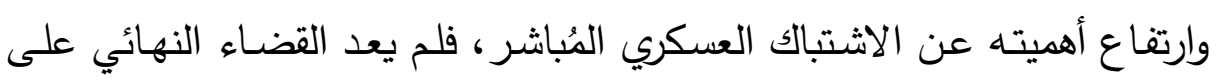

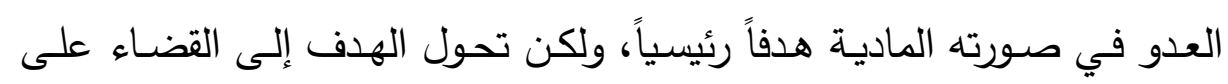

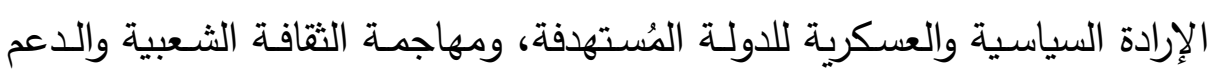


المدني للحرب منـذ البدايـة (39)، واتفاقـاً مـع هذا المبدأ تطورت الاسـتراتيجية الاستخباراتية لتواكب الدور الجديد الذي تلعبه لتحقيق أفضل النتائج لهذا الجيل الجديد من الحروب، حيث أصبح الاعتمـاد على شبكات التواصل الاجتماعي وعلى مواطني الدولة المُستهدفة أنفسهم (الاستخبار البشري) أكثر فاعلية منـه على الأقمار الصناعية العسكرية. 3- المنظمات الاولية غير الحكومية:

حيث تقوم حروب الجيل الرابع باستغلال عدداً من المنظمـات التي تُقدم الدعم والتدريب للشباب، ومنظمات المجتمع المدني داخل الدولة المُستهدفة لتنفيذ أجندات خارجية، من خلال تزويدها بالتمويل والتدريب لتأمين مصـالحها وتأكيد نفوذها، وترتبط هذه المنظمات بأنشطة سياسية واجتماعية وخدمية لتحقيق أكبر قدر من الاختراق مجتمعياً، بهدف زعزعة الاستقرار وهدم كيانات المجتمع من الداخل.

\section{4- النظام الحاكم نفسـه:}

قد يكون النظام الحاكم أحد أدوات حروب الجيل الرابع بإرادته، وهو إن وجد يُعد الأخطر على الإطلاق، فهذه الحروب تهدف إلى خلق دولة فاشلة وأن تصل إلى درجة من الانحلال يكون فيها آخر طرف باقي هو الحاكم ولا يكاد يستطيع الوقوف، وغالباً ما تتطبق تلك المخططات على التنظيمات التي تصل للسلكة

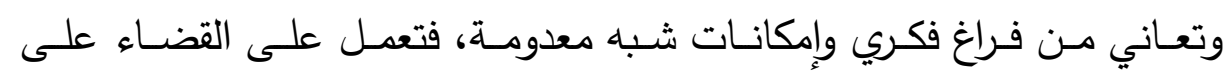


المسارات السياسية القائكـة لتجنب نهايتها المحتمـة خـارج إطـار السلطة ودوائر

النفوذ، خاصة إذا كان الإطار الفكري للنظام الحاكم لا يُحبذ فكرة الدولة (40).

\section{تاسعاً: أبعاد تأثير مخاطر حروب الحيل الرابع (41).}

تتعدد أبعاد تأثير حروب الجيل الرابع على أركان الدولة المُستهدفة وفي

كافة المجالات؛ ويتحلى أهمها فيما يلي:

1 - البُعد القومي، ويتمثل في:

تهديد الأمن القومي للمجتمع من خلال التشكيك في قدرات الشعب وقياداته، وطمس الهويـة الوطنيـة، وإفثـال نظـام الحكم والنظـام السياسـي في الدولـة، وزعزعة استقرارها ونشر الفوضى. 2- البُعد الإعلامي والتكنولوجي، ويتمثل في:

ترسيخ ثقافة الصورة والكلمة لدى الأفراد، وتوسيع النطاق لارتكاب الجرائم

$$
\begin{aligned}
& \text { الإلكترونية مثل التجس الإلكتروني، والإرهاب الإكتروني. } \\
& \text { 3- البُعد الثقافي والفكري، ويتمثل في: }
\end{aligned}
$$

تـدمير الثقافـة مـن خـلال تهديـــ القـيم الأصـيلة للمجتمـع مثل التماسـك الاجتماعي، والانتماء، والولاء، المسؤولية، بالإضـافة إلى الغزو الفكري للأفراد من خلال تزييف الوعي واستعماره، وغرس قيم وسلوكيات دخيلة على المجتمع. 


\section{4- الأبعد العسكري، ويتمثل في:}

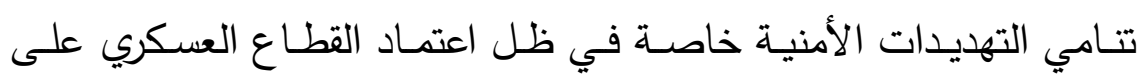
الوسائل التكنولوجية واتجاهه نحو الرقمنة، حيث أصبح ضرب القطاع العسكري

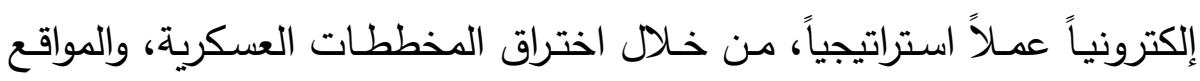
الإكترونية الحكومية والاطلاع على معلومات وبيانات الدولة المُستهدفة. 5- اليُعد الاجتماعي، ويتمثل في:

تزييف الوعي الاجتماعي، واختراق المجتهـع من الداخل، بهدف خلخلـة الهوية الوطنية ومحاولة تنكيك النسيج الهجتمعي القائم. 6- الثُعد الاقتصادي، ويتمثل في:

ضرب البنية التحتية للدولة المُستهدفة، واختراق المنظومة الاقتصادية من

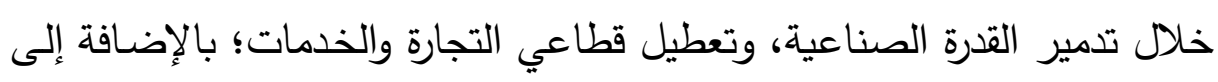
زيـادة الديونية، وارتفاع معدلات القروض الدولية، وتراجع الاستثمار المحلي لئي

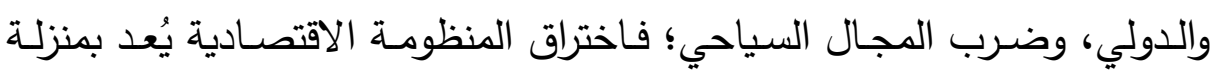
قطع لثريان الإنتاج، والتتوزيع، واستهلاك السلع، والخدمات. 


\section{الهوامش}

(1) آلاء ناصر حسين البعاج، فراس عبد المنعم عبد الله الثويلي، الحمايـة الجنائية لأمسن

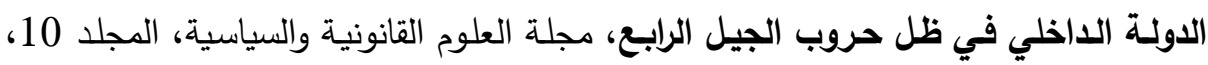

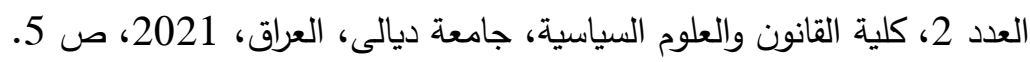

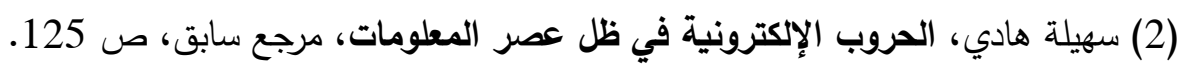

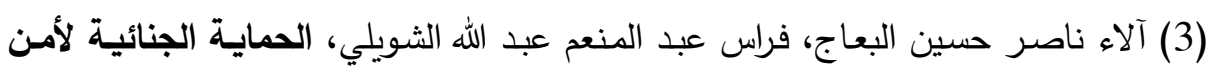

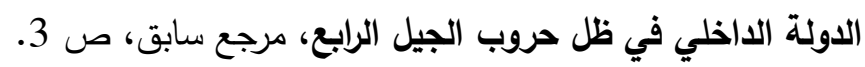
(4) Albert A. Nofi, "Recent Trends in Thinking about Warfare", The CNA Corporation, 2006, p. 8.

(5) شيماء الهواري، المنظومـة الإعلاميـة: الجيل الرابـع مـن الحروب والعمليات النفسية،

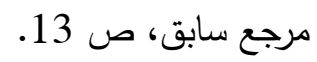

(6) William. S. Lind, Col Keith and Others "Understanding Fourth Generation War" Op. Cit, p. 12.

(7) شيماء الهواري، المنظومـة الإعلاميـة: الجيل الرابـ مـن الحروب والعمليـات النفسية،

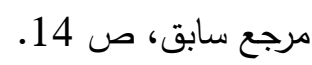

(8) William. S. Lind, Col Keith and Others "The changing Face of war: Into the Fourth Generation", Marine Corps Gazette, U.S.A., 1989, p. 22.

(9) آمال زرنيز ، أثر حروب الجيلين الرابع والخـامس في إدامـة الصراع بـدول المنطقـة

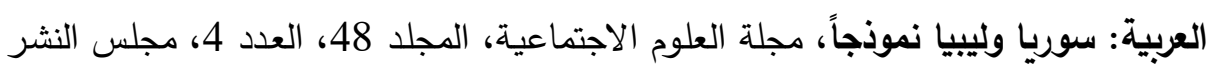

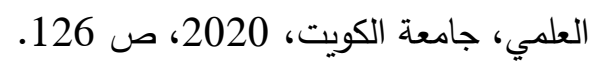

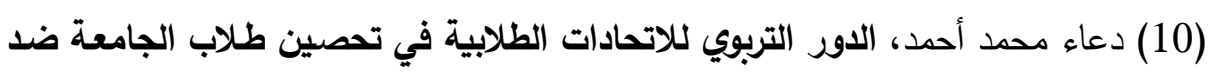

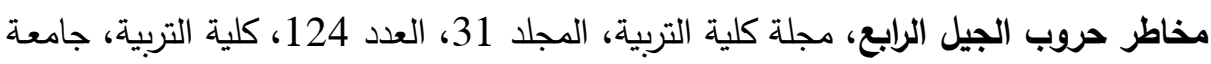

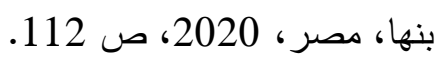

(11) نسرين حسـام الدين، دور مواقـع التواصـل الاجتمــاعي فـي إدراك الثـباب المصـري

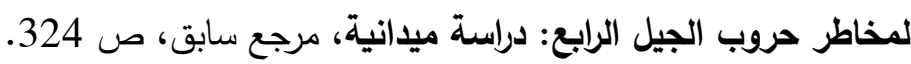

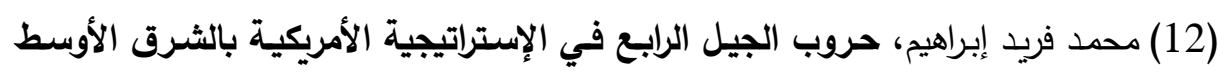

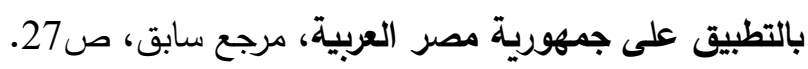

شيماء محمد محمد عرفة

(حروب الجيل الرابع: الآليات والأبعاد) 
(13) محمود محمد علي، حرب العصـابات وبدايـة بزوغ حروب الجيل الرابع، دار الوفاء

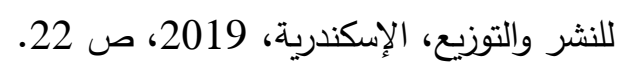

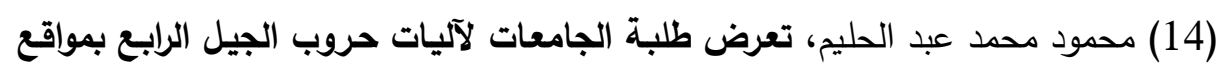

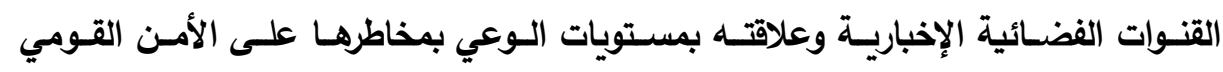

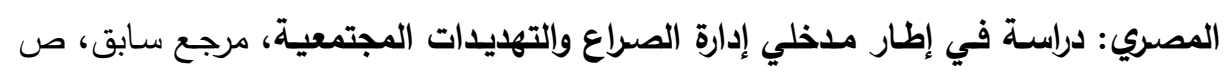

(15) Colonel Steven C. Williamson, "From Fourth Generation Warfare to Hybrid War", Op.Cit, p.3.

(16) سهيلة هادي، الحروب الإكترونية في ظل عصر المعلومات، مرجع سابق، ص 127.

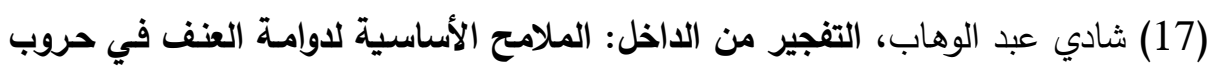

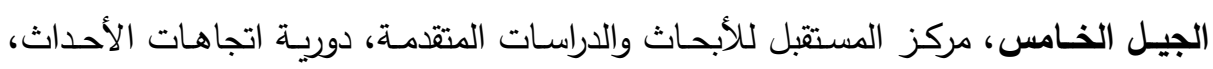

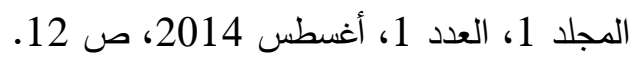

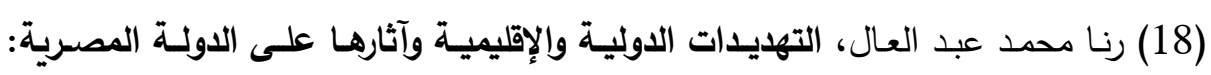
دراسة حالة حروب الجيل الرابع، مرجع سابق، ص صدال (19) زينب حسني عز الدين، أثر حروب الجيل الرابع على الأمس القومي العربي دراسيسة

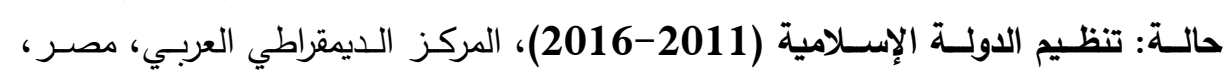
2016، ص صن 11.

(20) Thomas X. Hammes, "Insurgency: Modern warfare Evolves into a fourth generation" , Strategic Forum, No. 214, Institute for National Strategic Studies (INSS), National Defense University, U.S.A, Jan 2005, p. 5.

(21) محمود محمد علي، الفوضسى وزعزعـة الاستقرار وطبيعة حروب الجيل الرابع، دار

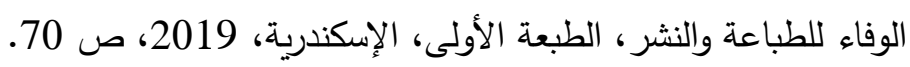

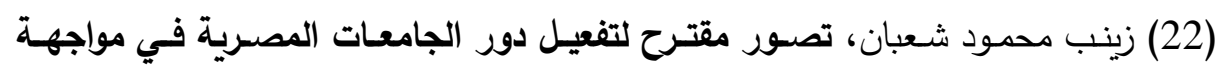

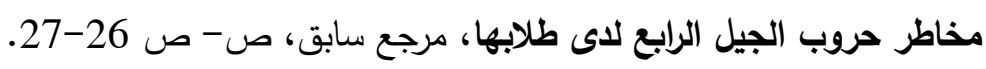

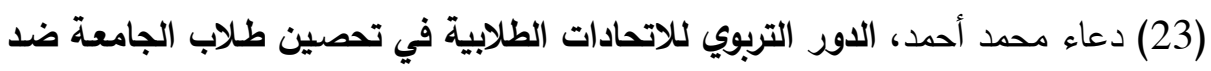

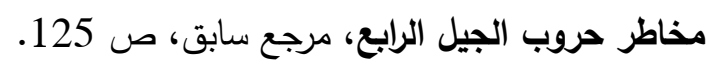

شيماء محمد محمد عرفة (حروب الجيل الرابع: الآليات والأبعاد) 
(24) Greg Simons, "Fourth Generation Warfare and Clash of

Civilizations", Journal of Islamic Studies, Vol. 21, Oxford University Press, UK, 2010, P. 412.

(25) جمال علي خليل الدهثان، الإرهاب في العصر الرقمي "الإرهاب الإكتروني": صوره،

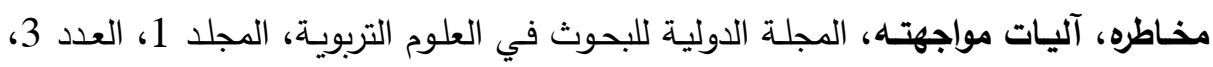
المؤسسة الدولية لآفاق المستقبل، إستونيا، 2018، صاتهات 95.

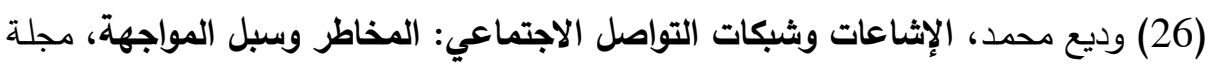

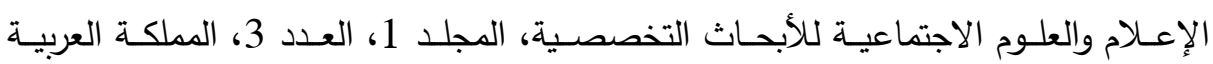

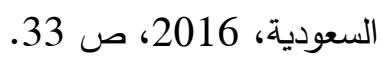

(27) سلوى أحمد ميدان، نهى عبد الخـالق أحمد، الثـائعات الإكترونيـة ودور المرجعيـة الدولية في التصدي لها، كلية القانون والعلوم السياسية، جامعة كركوك، العراق، (ب.تئ)، ص 6.

(28) آمنة حسين أحمد، الثـائعات كأحد وسائل الجيل الرابع في الحروب، المؤتمر العلمي

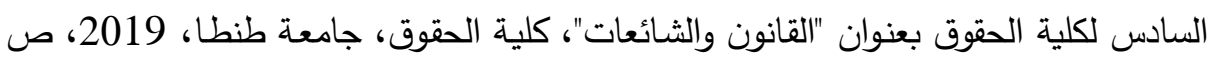

(29) شيماء الهواري، المنظومـة الإعلامية: الجيل الرابع من الحروب والعمليات النفسية،

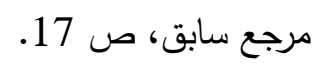

(30) ندية عبد النبي القاضي، اتجاهات النخبة المصرية نحو إدارة المواقع الإخباريـة لآليات حروب الجيل الرابع في مصر، مرجع سابق، ص لن 6.

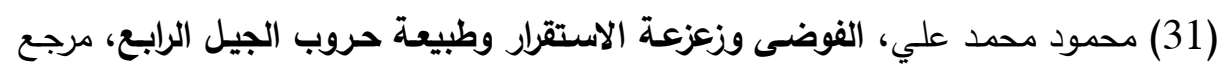
سابق، ص 76.

(32) إسلام عيسى، الأنثروبولوجيا ودورها في أجيال الحروب الحديثة، مرجع سابق، ص

(33) دعاء محمد أحمد، الاور التربوي للاتحادات الطلابية في تحصين طلاب الجامعة ضد

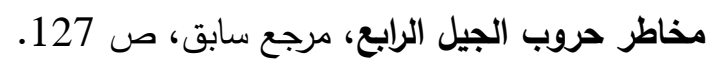

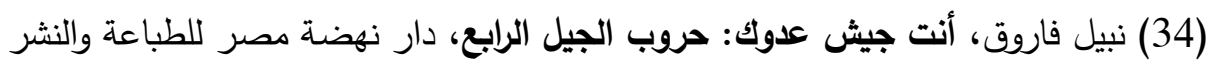
والتوزيع، مصر ، 2016، صن فاروق،

شيماء محمد محمد عرفة

(حروب الجيل الرابع: الآليات والأبعاد) 
(35) ريما موسى، دور وسائل الإعلام في حروب الجيل الرابع ومسؤوليتها في القانون

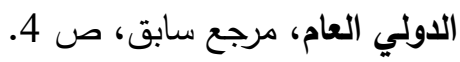

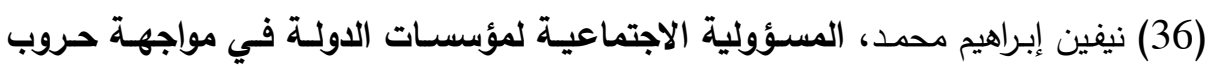

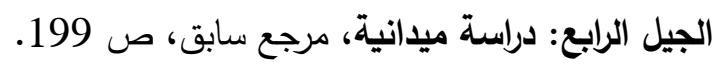

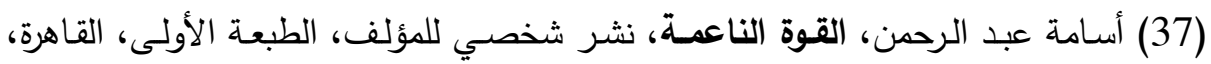
. 2018، صن 8

(38) Christina, M. Knopf, and Eric, J. Ziegelmayer, "Fourth generation warfare and the US Military's social media strategy", Op. Cit, p. 7.

(39) Edward Jamison, "Intelligence Strategy for Fourth Generation Warfare", U.S. Army War College, Carlisle, U.S.A, 2006, P. 44.

(40) نيفين إبراهيم محمد، المسؤولية الاجتماعيـة لمؤسسـات الدوبـة في مواجهة حروب

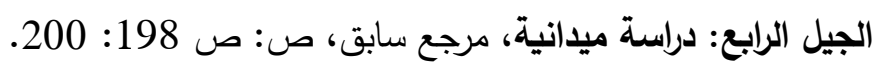

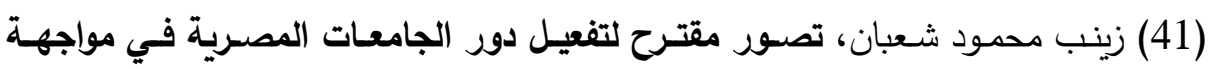

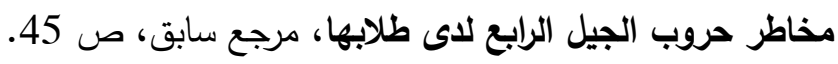

